\title{
Latest trends in imaging techniques for dental implant: a literature review
}

\begin{abstract}
During the past fewyears replacement of the missing teeth with dental implants has become an indispensible part of modern dental practice. As with other branches of dentistry for the optimal success in dental implant treatment proper diagnosis and treatment planning are the prerequisites. For proper diagnosis and treatment planning Implant imaging techniques and their accurate interpretation is a very crucial step. Radiography is the only non-surgical method of assessment of quality and the quantity of the bone at implant site. With the advent of several types of imaging techniques ranging from conventional two dimensional intraoral periapical radiographs and orthopantograph to highly sophisticated multidetector computed tomography (MDCT) and cone beam computed tomography (CBCT) selection of appropriate imaging technique for implant treatment planning has become a challenge for the clinicians. In this article latest imaging techniques used in implant dental practice are discussed along with their advantages and disadvantages in comparison with the conventional imaging techniques.
\end{abstract}

Keywords: implant imaging techniques, computed tomography, multidetector computed tomography
Volume 3 Issue 5 - 2017

\author{
Muhammad Waqar Hussain,' Muhammad \\ Aamir Ghafoor Chaudhary, ${ }^{2}$ Abdul Razzaq \\ Ahmed,' Shahabe Saquib Abullais ${ }^{3}$ \\ 'Department of Prosthodontics, King Khalid University, Saudi \\ Arabia \\ ${ }^{2}$ Department of Prosthodontics, Riphah International University \\ Islamabad, Pakistan \\ ${ }^{3}$ Department of Periodontics and Community Dental Sciences, \\ King Khalid University, Saudi Arabia
}

Correspondence: Shahabe Saquib Abullais, Department of Periodontics and Community Dental Sciences, King Khalid University, Abha, Asir, Saudi Arabia, Email drsaquin24@gmail.com

Received: May 28, 2017| Published: July 28, 2017
Abbreviations: MDCT, multidetector computed tomography; $\mathrm{CBCT}$, cone beam computed tomography; CT, computed tomography; PA, periapical radiographs; DPR, dental panoramic radiographs; TACT, tuned aperture computed tomography

\section{Introduction}

During the last couple of decades implant dentistry has evolved to become important part of modern dental practice. Until late 1980s dental implant treatment was restricted to edentulous patients only and was done at selected universities or specialist dental centers by a team of specialist dentists. ${ }^{1,2}$ Later with developments in implant material, design and components the dental implants found their application partially edentulous patients. ${ }^{3}$

Success of any dental treatment depends on accurate diagnosis and proper treatment planning. Diagnostic imaging techniques are important tool for treatment planning of implant prosthesis. ${ }^{2-4}$ Implant treatment planning requires radiographic examination of the implant site to ascertain information about the quality and the quantity of the bone available at the implant site and to accurately locate the position of any critical anatomical structure present in immediate vicinity of the implant site. Until late 1980s conventional radiographic techniques such as intraoral periapical radiographs, cephalometric and panoramic views were considered standards. With developments in radiography many more sophisticated cross-sectional radiographic imaging techniques like reformatted computerized tomography were introduced for implant dental treatment. In the year 2000, the American academy of oral and maxillofacial radiology specified that conventional cross-sectional tomography should be employed for the implant patients. ${ }^{5}$

Currently a wide variety of imaging techniques are available ranging from simple two dimensional conventional imaging techniques like intraoral periapical radiographs (PA) and dental panoramic radiographs (DPR) to highly accurate three dimensional imaging techniques like computed tomography (CT) and cone beam tomography (CBCT). Therefore selection of imaging technique for implant treatment planning has become a challenge. ${ }^{3,5}$ Dental panoramic radiographs have disadvantage of distortion and magnification and require radio opaque marker to correct distortion. On the other hand intra oral periapical radiograph is accurate but have the limitations in accurately locating critical anatomical structures like inferior alveolar canal. Computed tomography (CT) and cone beam tomography (CBCT) is highly accurate and give three dimensional images but have a drawback of higher radiation dose. In this article latest imaging modalities for dental implants will be discussed along with their advantages and disadvantages. ${ }^{4,5}$

\section{Tomography}

Term tomography originated from Greek language and is a combination of two words "Tomo" (Slice or section) and "Graph" (Picture). ${ }^{4,6}$ Devise used is called tomography and while the three dimensional image produced is called Tomogram. In This technique clear images of structures lying in one plan are obtained by blurring out the structures not in the desired plane. ${ }^{6}$

Conventional tomography is useful for single implant site or for multiple implants within one quadrant. Tomographic slices are crosssectional and are as thin as $1 \mathrm{~mm}$. Magnification of conventional tomographic images is constant in all directions therefore measurements can be made using a special ruler provided with appropriate scale or measurements can be made using a measurement programme in case of digital images. ${ }^{7}$ Conventional tomography is not of much help in determining the difference in most bone densities or identifying any disease at implant site. Tomography is highly technique sensitive with superimposition of surrounding structure results in blurring of the image. ${ }^{8}$ Diagnostic quality of tomographic images is determined by the type of tomographic motion, section thicknes and the degree of 
magnification. Different methods have been devised like spiral and linear to reduce the blurring artifacts. ${ }^{8-10}$

Linear tomography is the simplest form of tomography but it results in typical streak artifacts called 'parasite lines'. ${ }^{9}$ Tomographic images have constant magnification that depends on the distance between the focus to film and film to object. Any metallic restoration adjacent to desired area may distort the desired image. ${ }^{10}$

\section{Spiral tomography}

In spiral tomography topographic images are produced using spiral movement and blurred shadows are kept at equal distances from each other. Spiral tomography gives better 3D contrast and spatial resolution. ${ }^{11}$ A series of four images are produced with a fixed projection angle, each of $4 \mathrm{~mm}$ thickness and $4 \mathrm{~mm}$ apart from each other. Each fill shows $16 \mathrm{~mm}$ thick section of maxilla or mandible. Magnification for spiral tomography varies from 10-30\%, higher the magnification better will be the quality of image. Spiral tomography can be used to determine the spatial relationship between critical structures and the implant site. ${ }^{11-13}$

\section{Transtomography or sectional tomography}

In this technique digital transtomographic images are produced by combining the translational movement with the pendular movement of the beam and detector. ${ }^{12}$ This technique is helpful in establishing spatial relationship between critical structures and the implant site but cannot be used for determining the differences in most bone densities or to identify any disease at implant site. This technique can be used for individual implant site only because the adjacent structures that is blurred and superimposed on the image. ${ }^{11-13}$

\section{Computed tomography (CT)}

Housefield a British engineer invented CT in 1972.Computed tomography was originally developed for examination of soft tissues particularly brain. It is an advanced imaging technique in which cross-sectional tomographic images are obtained digitally and later image data can be reformatted into any plane such as axial sagittal or coronal. ${ }^{7,11,13}$ A narrow $\mathrm{x}$-ray beam is used to scan the patient at right angle to the long axis of the body and resulting axial images are thin $1-2 \mathrm{~mm}$. With advancement of CT, the reformatted images can be seen with a section thickness of 1 pixel and an in-plane resolution of 1 pixel by the scan spacing $0.5-1.5 \mathrm{~mm}$ and produce a geometric resolution similar to that of planar imaging. ${ }^{6}$ Density of structures within the image is absolute and can be used to differentiate tissues in the image. Computed tomography useful for the identification of proximity of the vital structures and the disease at implant site. ${ }^{12,14}$

Tissues which differ in physical density by less than one percent can be distinguished without super imposition computed tomography can produce 3 dimensional images with high resolution and uniform magnification. ${ }^{9,12}$ When compared with peri-apical and panoramic radiographs computed tomography can accurately locate mandibular canal. Three dimensional reconstructions are possible with computed tomography. Main disadvantage of computed tomography is the high cost and higher dose of radiation absorbed by the patient in comparison with the dose administered during panoramic and linear tomography. ${ }^{13,14}$

\section{Cone beam tomography (CBCT)}

Cone beam tomography is highly advanced form computed tomography. It produces 3 dimensional images of high resolution with low cost and radiation dosage. Image data obtained can be reformatted using software for customized visualization of anatomy. ${ }^{7,8}$ Cone beam tomography is used for wide range of craniofacial indications such as jaw bone lesions. A cone shaped ray of ionizing radiation is directed through the middle of desired area onto an X-ray detector. Cone beam tomography has wide range of applications in dentistry such as dental traumatology, apical surgery, challenging periodontal bone defects, endodontics, orthodontics, preoperative planning of periodontal surgery, forensic odontology and dental implant surgery. With CBCT 3D multi planner visualization of maxillofacial skeleton is possible and the image volume can be reconstructed in any plane. $\mathrm{CBCT}$ in implant dentistry is used for quantitative and qualitative bone assessment and it can be used for detection of bone disease at implant site. ${ }^{11,13}$

Studies comparing the reliability of CBCT with CT have confirmed the superiority of $\mathrm{CBCT}$ over spiral $\mathrm{CT}$ in terms of resolution of cross-sectional image. Main advantages of $\mathrm{CBCT}$ is reduced size and cost of CT scanner, better resolution of image with reduced dose of radiation as compared with conventional CT scan. ${ }^{5}$ Radiation dose for $\mathrm{CBCT}$ ranges from 2 to 8 panoramic radiographs much less than conventional Computed tomography. In comparison with periapical radiography and panoramic radiography $\mathrm{CBCT}$ is free of image distortion and magnification. Therefore with CBCT quantity of bone in width and height at implant site can be measured with high level of accuracy. Quality of bone, thickness of cortical plate, trabecular pattern and relationship of any vital structure such as inferior alveolar nerve can be assessed exactly at implant site with CBCT. There are several disadvantages of CBCT including radiation scattering, long scanning time and limited dynamic range of the X-ray area detectors. ${ }^{15}$

\section{Tuned aperture computed tomography (TACT)}

Tuned Aperture computed tomography TACT is a new technique as an alternative to film based Computed tomography. ${ }^{4,7}$ In this technique image is produced by passing a radiographic beam through an object several different angles. TACT uses several small radiographic tubes which are fired in close sequence and produce a single 3 dimensional image from the data collected by the sensor. ${ }^{7}$ Advantage of TACT is, it can accommodate patient movement during firing with affecting the quality of the image produced with reduced radiation dose. Localization of bone disease, anatomical structures and abnormalities at implant site is much easier with TACT. In dentistry TACT is being used to identify crestal defect around implant in addition to early detection of subtle decay. ${ }^{11,14}$

\section{Interactive CT}

Most significant advancement in CT is interactive computed tomography ICT. ICT allow the transfer of the information from radiologist to practitioner in form of a computer file. Practitioner uses specialized software to measure the width and the height of the bone and to measure bone quality at implant site..$^{13,15}$

In Interactive CT a 3D treatment plan integrated with patient's anatomy is devised and clinician performs electronic surgery on computer before actual implant surgery. ${ }^{11,15}$ Interactive CT allow the clinician to measure proximity of any vital structure to the implant site. Interactive tomography requires specialized software for interactive analysis and treatment planning on the basis of information received from radiologist in the form of computer file. ${ }^{8}$ Most important feature of ICT is that Position of prosthesis can be translated to scan and this 
allows the placement of implants dictated by the prosthesis during electronic surgery. Data collected in this way is used to fabricate a surgical guide that allows the accurate placement of dental implants at the time of actual implant surgery. ${ }^{8,9}$

\section{Denta-scan imaging}

Denta scan is new computer software which produces Computed tomography images of mandible and maxilla in three planes of reference: axial, panoramic and oblique sagittal. These 3D Images allow the accurate presurgical treatment planning. Denta-scan has the advantage of allowing evaluation of bone height and width, identification of pathology and location of anatomical structure. ${ }^{6,8}$ Disadvantage of Denat- scan is radiation exposure, increased cost and image magnification because the images produced are not of true size and require compensation for magnification.

\section{Magnetic resonance imaging}

Magnetic resonance imaging or MRI is of recent origin in the field of dental implantology. MRI is used when soft tissue imaging is required such as location of inferior dental canal. Sometimes MRI is used as secondary imaging technique when primary imaging technique like CT or ICT fail to differentiate inferior alveolar canal or neurovascular bundle from the adjacent trabecular bone carrying fat in trabecular bone. ${ }^{8,9}$ Studies have shown that geometric accuracy of MRI is compareable to CT and it is an acceptable imaging modality for dental Implantology. ${ }^{11,12}$

Ferromagnetism is the phenomenon related to MRI which results in production of artifacts in the image if the components of implant are ferromagnetic in nature. This is the reason which limits the use of MRI as an imaging modality after implant placement. ${ }^{13}$ Exposure of dental implant to MRI may result in heating of implant which may interfere with osseointegration. ${ }^{12}$ Limiting factor in the use of MRI for implantology is its limited ability in detecting any bone or dental disease and the bone mineralization at implant site. ${ }^{14,15}$

\section{Conclusion}

There are many imaging modalities available for imaging the implant site in the present day situation; however, the correct and required technique should be embraced depending on the type of case and the clinician's judgment. The choice of implant imaging must be evaluated carefully due to the exposure dose, the high cost of various technique and information that may be provided by the imaging study. The risk-to-benefit ratio should be evaluated depending on the case to maximize success.

Conventional radiographic technique is of little importance in implant imaging, still for many clinicians it is the technique of choice due to its cost effectiveness. MRI can be used to access the exact locations of vital structures. It is best used when the detection and evaluation of soft tissue is required. Quantitative and qualitative analyses of bone can be conducted at implant site by using CT scan. Construction of a surgical template is possible with the introduction of software used with CT or MRI. The recent technology used for dental implant imaging is $\mathrm{CBCT}$, as it provides the rapid acquisition of data with little radiation exposure.

\section{Acknowledgements}

None.

\section{Conflict of interest}

Author declares that there is no conflict of interest.

\section{References}

1. Mozzo P, Procacci C, Tacconi A, et al. A new volumetric CT machine for dental imaging based on the conebeam technique: preliminary results. Eur Radiol. 1998;8(9):1558-1564.

2. Academy of Osseointegration. 2010 Guidelines of the Academy of Osseointegration for the provision of dental implants and associated patient care. Int J Oral Maxillofac Implants. 2010;25(3):620-627.

3. Benavides E, Rios HF, Ganz SD, et al. Use of cone beam computed tomography in implant dentistry: the International Congress of Oral Implantologists consensus report. Implant Dent. 2012;21(2):78-86.

4. Harris D, Buser D, Dula K, et al. E.A.O. guidelines fof the use of diagnostic imaging in implant dentistry. A consensus workshop organized by the European Association for Osseointegration in Trinity College Dublin. Clin Oral Implants Res. 2002;13(5):566-570.

5. Harris D, Horner K, Gröndahl K, et al. E.A.O. guidelines for the use of diagnostic imaging in implant dentistry 2011. A consensus workshop organized by the European Association for Osseointegration at the Medical University of Warsaw. Clin Oral Implants Res. 2012;23(11):1243-1253.

6. Mercier P, Lafontant R. Residual alveolar ridge atrophy: classification and influence of facial morphology. J Prosthet Dent. 1979;41(1):90-100.

7. Seibert JS, Salama H. Alveolar ridge preservation and reconstruction. Periodontol. 1996;11:69-84.

8. Tallgren A. The continuing reduction of the residual alveolar ridges in complete denture wearers: a mixedlongitudinal study covering 25 years. J Prosthet Dent. 1972;27(2):120-132.

9. Lofthag-Hansen S, Grondahl K, Ekestubbe A. Cone-beam CT for preoperative implant planning in the posterior mandible: visibility of anatomic landmarks. Clin Implant Dent Relat Res. 2009;11(3):246-255.

10. Quirynen M, Mraiwa N, van Steenberghe D, et al. Morphology and dimensions of the mandibular jaw bone in the interforaminal region in patients requiring implants in the distal areas. Clin Oral Implants Res. 2003;14(3):280-285.

11. Jacobs R, Mraiwa N, vanSteenberghe D, et al. Appearance, location, course, and morphology of the mandibular incisive canal: an assessment on spiral CT scan. Dentomaxillofac Radiol. 2002;31(5):322-327.

12. State of the Science on Implant Dentistry. Consensus conference proceedings. Int J Oral Maxillofac Implants. 2006. p. 7-226.

13. Guler AU, Sumer M, Sumer P, et al. The evaluation of vertical heights of maxillary and mandibular bones and the location of anatomic landmarks in panoramic radiographs of edentulous patients for implant dentistry. $J$ Oral Rehabil. 2005;32(10):741-746.

14. Bennett DA. Computed tomography: Reducing the Cost and Increasing the Effectiveness of Dental Implantology. Dent Implantol Update. 2005;16(8):57-63.

15. Dreiseidler T, Mischkowski RA, Neugebauer J, et al. Comparison of cone-beam imaging with orthopantomography and computerized tomography for assessment in presurgical implant dentistry. Int J Oral Maxillofac Implants. 2009;24(2):216-225. 